\title{
Identification of a DNA methylation marker that detects the presence of lymph node metastases of gastric cancers
}

\author{
YASUYUKI SHIGEMATSU ${ }^{1}$, TOHRU NIWA ${ }^{1}$, SATOSHI YAMASHITA $^{1}$, \\ HIROKAZU TANIGUCHI ${ }^{2}$, RYOJI KUSHIMA ${ }^{2}$, HITOSHI KATAI $^{3}$, SEIJI ITO ${ }^{4}$, \\ TETSUYA TSUKAMOTO $^{5}$, MASAO ICHINOSE $^{6}$ and TOSHIKAZU USHIJIMA $^{1}$ \\ ${ }^{1}$ Division of Epigenomics, National Cancer Center Research Institute, Chuo-ku, Tokyo 104-0045; \\ Divisions of ${ }^{2}$ Pathology and Clinical Laboratory and ${ }^{3}$ Gastric Surgery, National Cancer Center Hospital, Chuo-ku, \\ Tokyo 104-0045; ${ }^{4}$ Department of Gastroenterology, Aichi Cancer Center Hospital, Chikusa-ku, Aichi 464-8681; \\ ${ }^{5}$ Department of Diagnostic Pathology, School of Medicine Fujita Health University, Kutsukake-cho, Toyoake, Aichi 470-1192; \\ ${ }^{6}$ Second Department of Internal Medicine, Wakayama Medical University, Kimiidera, Wakayama 641-8509, Japan
}

Received December 8, 2011; Accepted May 3, 2012

DOI: $10.3892 / \mathrm{ol} .2012 .708$

\begin{abstract}
The accurate detection of the presence of lymph node metastases (LNM) of gastric cancers (GCs) is useful for the implementation of necessary and sufficient treatment, but current methods of detection are unsatisfactory. In the present study, we focused on DNA methylation markers since they have several advantages, including biological and chemical stability and informativeness even in the presence of contaminating cells. Using three metastatic lymph nodes and three primary GCs without LNM, methylation bead array analyses were performed, which enabled the interrogation of 485,577 CpG sites. A total of $31 \mathrm{CpG}$ sites that were hypermethylated in the metastatic lymph nodes, compared with the GCs without LNM, were isolated. Using primary GCs with and without LNM (28 GCs with LNM and 10 without), their methylation levels were measured using quantitative PCR following treatment with sodium bisulfite or a methylationsensitive restriction enzyme. Of the genomic regions around the $31 \mathrm{CpG}$ sites, 10 regions demonstrated higher methylation levels in the GCs with LNM compared with the GCs without LNM $(\mathrm{P}<0.05)$. Finally, the hypermethylation of the 10 regions was validated using another set of samples (129 GCs with LNM and 20 without). Hypermethylation of the region around the cg06436185 CpG site predicted the presence of LNM at a sensitivity of $43 \%$ and specificity of $85 \%$. Additionally, the hypermethylation of the region was associated with a poor survival rate among GC patients with LNM. The results of
\end{abstract}

Correspondence to: Dr Toshikazu Ushijima, Division of Epigenomics, National Cancer Center Research Institute, 1-1 Tsukiji 5-chome, Chuo-ku, Tokyo 104-0045, Japan

E-mail: tushijim@ncc.go.jp

Key words: DNA methylation, gastric cancer, lymph node, metastasis the present study indicated that the methylation status of the region was a promising candidate marker to detect the presence of LNM of GCs and may reflect the malignant potential of GCs.

\section{Introduction}

Gastric cancer (GC) is one of the most prevalent malignancies worldwide and remains a leading cause of cancer-related mortality $(1,2)$. Since the presence of lymph node metastases (LNM) is associated with a significantly poorer prognosis of GC patients (3-5), radical resection with free-margin gastrectomy and extended lymphadenectomy are performed for patients with advanced GC to eradicate LNM (6). Such an aggressive resection of the lymph nodes is associated with higher patient morbidity and/or mortality rates (7-9). Alternatively, the absence of LNM allows for minimally invasive surgery, which provides an improved quality of life following treatment. Therefore, the accurate detection of LNM is useful for the implementation of necessary and sufficient treatment.

To detect the presence of LNM, much effort has been made in the fields of imaging and molecular markers. Imaging modalities, including computed tomography (CT), endoscopic ultrasonography (EUS) and ${ }^{18} \mathrm{~F}$-fluorodeoxyglucose positron emission tomography (FDG-PET) are used in clinical practice. However, the sensitivities of these modalities are 77.2, 82.8 and $71 \%$, respectively, and the specificities are 78.3, 74.2 and $74 \%$, respectively (10-13). Moreover, these imaging modalities are almost powerless to detect micrometastases $(14,15)$. With regard to molecular markers, analyses that targeted specific RNA and protein expression have been made. Although a number of these markers were associated with the presence of LNM of GCs (16-19), their utility has not been confirmed by independent studies. Therefore, genome-wide or comprehensive analysis of molecular markers for LNM of GCs is required and validation of the utility of the markers is essential for clinical application. 
As a molecular marker, DNA methylation is advantageous, as its status is stable even if a cell is placed in different environments (biologically stable) and DNA is chemically stable, even in clinical materials. In addition, DNA methylation profiles are not disturbed by the presence of a small population of contaminating cells. As a strategy, we used metastatic lymph nodes and primary GCs without LNM for genome-wide analysis as cells with the abililty of LNM may constitute only a small population of the cells in primary GCs with LNM. Differences in methylation levels may be extremely small and may not be detected by the analysis between primary GCs with and without LNM. Alternatively, in metastatic lymph nodes, cancer cells are expected to possess the aberrant DNA methylation following clonal selection. Moreover, the methylation levels of appropriate marker $\mathrm{CpG}$ sites in the metastatic lymph nodes are expected to be relatively high compared with those in primary GCs with LNM.

In the present study, we aimed to identify $\mathrm{CpG}$ sites with a methylation status associated with the presence of LNM of GCs via a genome-wide methylation analysis using metastatic lymph nodes and primary GCs without LNM and to validate the isolated candidate markers.

\section{Materials and methods}

Patients, tissue samples and DNA extraction. A total of 187 GC surgical samples were obtained from patients who underwent gastrectomy with extended lymph node dissection (D2) at the National Cancer Center Hospital (Tokyo, Japan) and Aichi Cancer Center Hospital (Aichi, Japan) between 1994 and 2011 with informed consent. A total of three metastatic lymph nodes were obtained from 3 of the 187 patients. No patients had undergone prior chemotherapy or radiotherapy. Prognostic information of 55 GC patients with LNM was available and the mean follow-up period after surgery was 3,024 days. Disease grades were classified according to the 6th edition of the TNM classification by the UICC. Samples were stored at $-80^{\circ} \mathrm{C}$ and a high molecular weight DNA was extracted using the phenol/chloroform method. The 187 samples were divided into screening (28 GCs with LNM and 10 without) and validation (129 GCs with LNM and 20 without) sets in advance, between which no significant differences in clinicopathlogical data were observed (Table I). This study was conducted with the approval of the Aichi Cancer Center and National Cancer Center.

Genome-wide methylation analysis. Genome-wide screening of differentially methylated $\mathrm{CpG}$ sites was performed using an Infinium HumanMethylation450 BeadChip array, which covers 485,577 CpG sites (Illumina, San Diego, CA, USA) (20). Genomic DNA $(1 \mu \mathrm{g})$ was treated with sodium bisulfite using a Zymo EZ DNA Methylation kit (Zymo Research, Irvine, CA, USA) and the bisulfite-modified DNA was amplified prior to hybridization to the array. The array was scanned with an iScan System (Illumina) and the data were analyzed using GenomeStudio Methylation Module Software (Illumina). A $\mathrm{CpG}$ site was considered to be informative if the sum of the signals for methylated and unmethylated sequences at the $\mathrm{CpG}$ site was significantly higher $($ at $\mathrm{P}<0.05)$ than signals of the negative control probes on the same array. Methylation levels were represented by $\beta$ values, with a $\beta$ value of 0 corresponding to no methylation and 1 corresponding to full methylation.

Quantitative methylation-specific PCR (qMSP). Sample DNA was treated with sodium bisulfite and purified as described previously (21). qMSP was performed using real-time PCR with bisulfite-modified DNA and specific primers (Table II, Fig. 1A). A methylation level was expressed as a percentage of the value of methylated DNA reference (PMR) calculated as the [(number of fragments methylated at a target locus in sample/number of the Alu sequences in sample)/(number of fragments methylated at a target locus in SssI-treated DNA/ number of the Alu sequences in SssI-treated DNA)]x100 (22).

Quantitative PCR following treatment with a methylationdependent restriction enzyme (qPTMR). A fully unmethylated control was prepared by amplifying human blood genomic DNA with phi29 DNA polymerase (Illustra GenomiPhi HY kit, GE Healthcare, Buckinghamshire, UK) (23). DNA (1 $\mu \mathrm{g})$ was treated with MspJI (New England Biolabs, Beverly, MA, USA), which cleaves DNA 9 bp downstream from the ${ }^{\mathrm{m}} \mathrm{CNNR}$ sequence $(24,25)$, in a $30 \mu \mathrm{l}$ reaction [4 $\mathrm{U}$ of $M s p J I, 1 \mathrm{X}$ NEB buffer 4 (New England Biolabs) and $0.1 \mathrm{mg} / \mathrm{ml} \mathrm{BSA}$ ] at $37^{\circ} \mathrm{C}$ for 20 h. Following purification, the DNA was treated with MspJI again and dissolved in TE $(10 \mathrm{mM}$ Tris- $\mathrm{HCl} \mathrm{pH} 8.0,1 \mathrm{mM}$ EDTA) at a concentration of $5 \mathrm{ng} / \mu \mathrm{l}$ without purification. Using $1 \mu \mathrm{l}$ of the solution, quantitative PCR (qPCR) was performed by real-time PCR with primers that encompassed a target $M s p \mathrm{JI}$ site (Fig. 1B). To normalize the quantity of input DNA, the number of copies of a standard sequence, which may be amplified with a primer pair (5'-TTGCTTGAAGTTTTGTTGCTGTAGT-3' and 5'-AATAAACTCAGTTGTGACATGGACA-3') and contains no MspJI site, was measured by qPCR. A percentage of the value of unmethylated reference (PUR) was calculated as the [(number of fragments at target locus in sample/number of the standard sequence in sample)/(number of fragments at target locus in GenomiPhi-amplified DNA/number of the standard sequences in GenomiPhi-amplified DNA)]x100. For convenience, the methylation level was expressed as 100-PUR.

Statistical analysis. Statistical analyses were conducted using PASW statistics version 18.0.0 (SPSS Japan Inc., Tokyo, Japan). The difference between the mean values of the two groups of samples was evaluated using Welch's t-test. The Fisher's exact test was used to evaluate the significant difference in relative frequency of the phenomena between two independent groups. Survival curves were computed according to the KaplanMeier method and the log-rank test was employed to evaluate the level of significant difference. $\mathrm{P}<0.05$ was considered to indicate a statistically significant difference.

\section{Results}

Genome-wide screening using metastatic lymph nodes and GCs without LNM. To isolate the CpG sites that are hypermethylated specifically in GCs with LNM, genome-wide methylation analysis was performed using metastatic lymph nodes $(n=3)$ and GCs without LNM $(n=3)$ using an Infinium HumanMethylation450 BeadChip array. The samples used for this analysis were prepared from 6 patients in the screening 
Table I. Clinicopathological data of sample sets.

$\mathrm{N} \quad$ Age (years) P-value Gender N P-value T stage N P-value

Genome-wide analysis set ${ }^{\mathrm{a}}$

\begin{tabular}{|c|c|c|c|c|c|c|c|c|c|}
\hline \multirow[t]{4}{*}{ Meta (-) } & 3 & $72 \pm 4$ & 0.17 & Male & 2 & 1.0 & $\mathrm{~T} 1$ & 0 & 0.51 \\
\hline & & & & & & & $\mathrm{T} 2$ & 1 & \\
\hline & & & & Female & 1 & & T3 & 1 & \\
\hline & & & & & & & $\mathrm{T} 4$ & 1 & \\
\hline \multirow[t]{4}{*}{ Meta $(+)$} & 3 & $59 \pm 13$ & & Male & 2 & & $\mathrm{~T} 1$ & 0 & \\
\hline & & & & & & & $\mathrm{T} 2$ & 0 & \\
\hline & & & & Female & 1 & & T3 & 1 & \\
\hline & & & & & & & $\mathrm{T} 4$ & 2 & \\
\hline \multicolumn{10}{|c|}{ Screening set } \\
\hline \multirow[t]{4}{*}{ Meta (-) } & 10 & $69 \pm 6$ & 0.13 & Male & 7 & 0.53 & $\mathrm{~T} 1$ & 0 & 0.17 \\
\hline & & & & & & & $\mathrm{T} 2$ & 1 & \\
\hline & & & & Female & 3 & & $\mathrm{~T} 3$ & 6 & \\
\hline & & & & & & & $\mathrm{T} 4$ & 3 & \\
\hline \multirow[t]{4}{*}{ Meta $(+)$} & 28 & $63 \pm 11$ & & Male & 18 & & $\mathrm{~T} 1$ & 0 & \\
\hline & & & & & & & $\mathrm{T} 2$ & 0 & \\
\hline & & & & Female & 10 & & $\mathrm{~T} 3$ & 14 & \\
\hline & & & & & & & $\mathrm{T} 4$ & 14 & \\
\hline \multicolumn{10}{|c|}{ Validation set } \\
\hline \multirow[t]{4}{*}{ Meta (-) } & 20 & $63 \pm 11$ & 0.71 & Male & 13 & 0.6 & $\mathrm{~T} 1$ & 0 & 0.14 \\
\hline & & & & & & & $\mathrm{T} 2$ & 3 & \\
\hline & & & & Female & 7 & & T3 & 8 & \\
\hline & & & & & & & $\mathrm{T} 4$ & 9 & \\
\hline \multirow[t]{4}{*}{ Meta (+) } & 129 & $62 \pm 10$ & & Male & 91 & & $\mathrm{~T} 1$ & 0 & \\
\hline & & & & & & & $\mathrm{T} 2$ & 4 & \\
\hline & & & & Female & 38 & & T3 & 55 & \\
\hline & & & & & & & $\mathrm{T} 4$ & 70 & \\
\hline
\end{tabular}

${ }^{a}$ This set comprised samples from the screening set.

$\mathbf{A}$

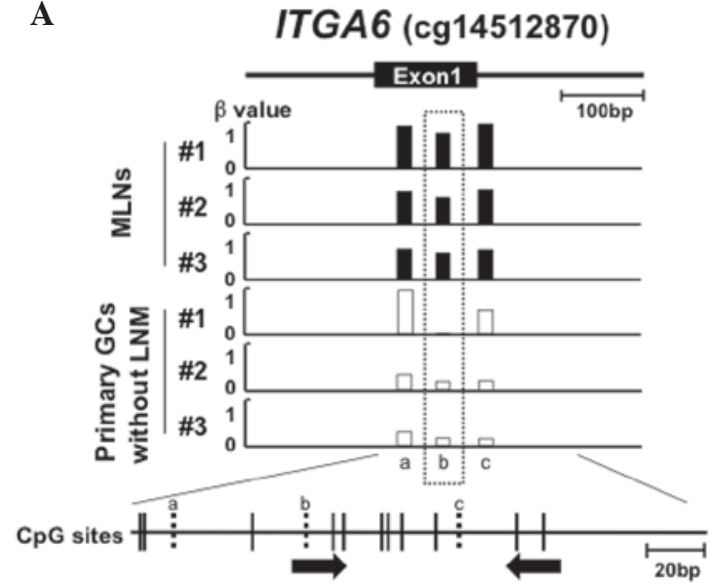

B

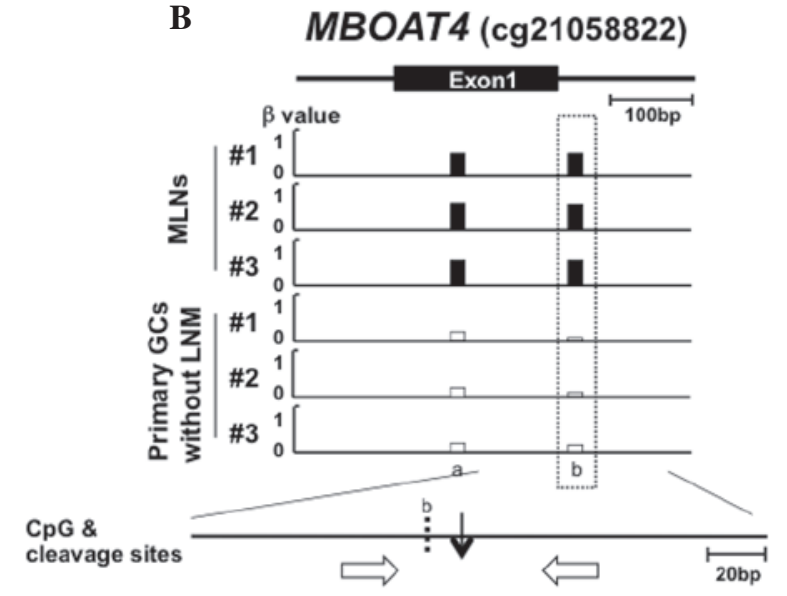

Figure 1. Representative genomic regions around the CpG sites differentially methylated between metastatic lymph nodes and GCs without LNM and primer design in the regions. Below the genomic structure of a region, $\beta$ values (methylation levels) of the CpG sites carried by Infinium bead array are shown. The differentially methylated CpG site is marked by a rectangle with dotted line. A CpG map is drawn at the bottom, vertical lines (solid and broken lines) indicate $\mathrm{CpG}$ sites and broken lines indicate $\mathrm{CpG}$ sites whose $\beta$ values were measured. (A) A region whose methylation level was assessed by qMSP. Primers specific to the methylated sequence (closed arrows) were designed on $\mathrm{CpG}$ sites around the differentially methylated sites based on the bisulfite-modified sequence. (B) A

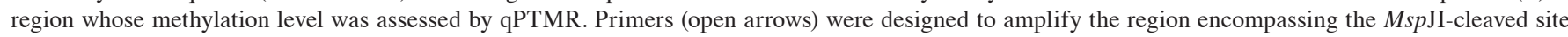
(thin vertical arrow) based on the unmodified sequence. GC, gastric cancer; MLN, metastatic lymph nodes; LNM, lymph node metastases; qMSP, quantitative methylation-specific PCR; qPTMR, quantitative PCR following treatment with a methylation-dependent restriction enzyme. 


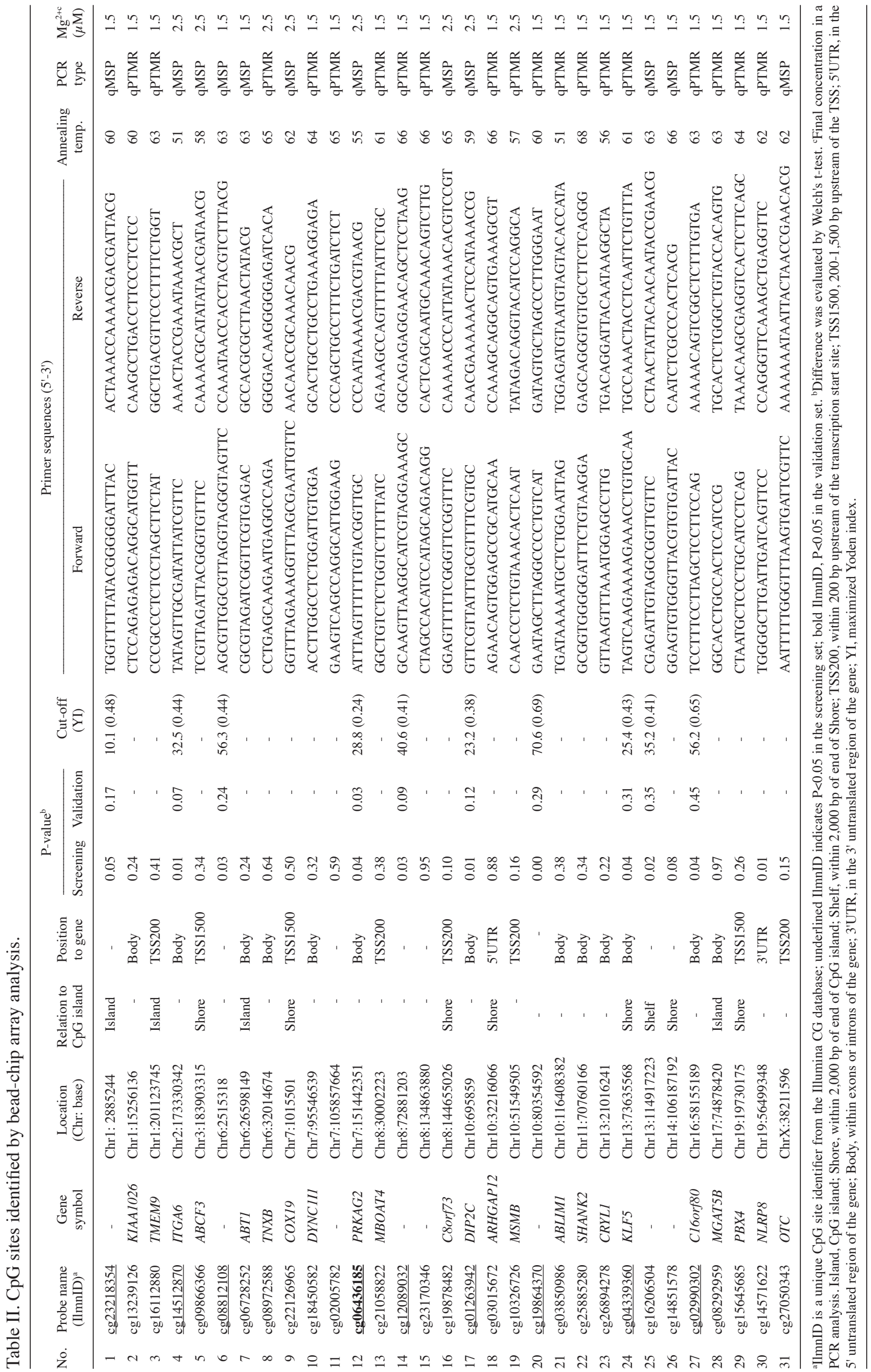


A
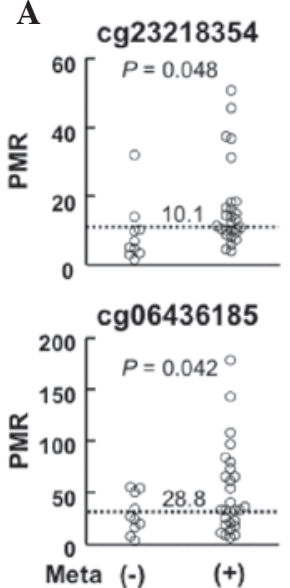

B
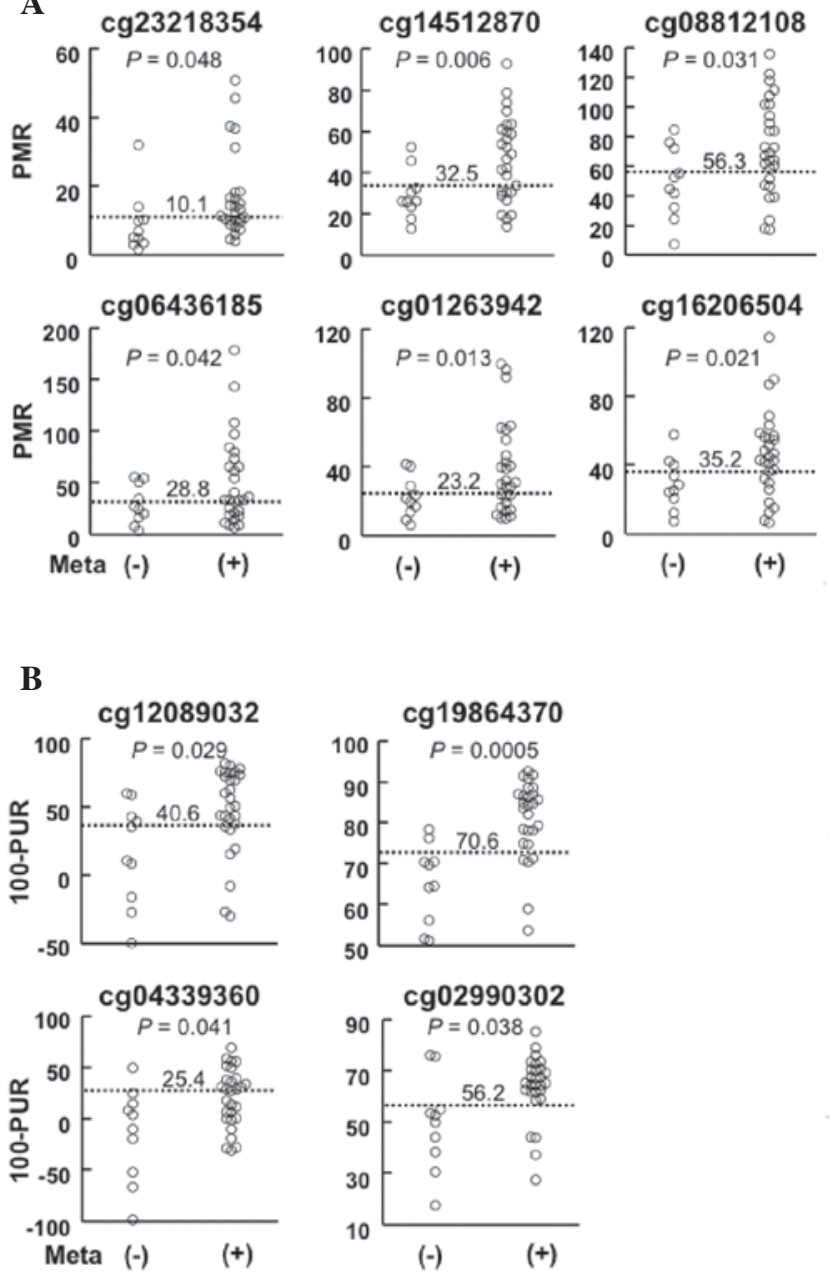

C

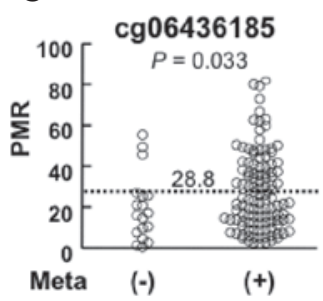

Figure 2. Methylation levels of the candidate genomic regions in primary GCs with and without LNM. Methylation levels were measured by (A) qMSP and (B) qPTMR in the screening sets. The screening set consisted of $10 \mathrm{GCs}$ without LNM and 28 with LNM. (C) Methylation level of the region around $\operatorname{cg} 06436185$ in the validation sets was measured by qMSP. The validation set consisted of 20 GCs without LNM and 129 with LNM. Meta (-), GCs without LNM; Meta (+), those with LNM. Horizontal dotted lines are the cut-off methylation levels and the number on the line indicates the value of the level. GC, gastric cancer; LNM, lymph node metastases; qMSP, quantitative methylation-specific PCR; qPTMR, quantitative PCR following treatment with a methylation-dependent restriction enzyme; PUR, percentage of the value of unmethylated reference; PMR, percentage of the value of methylated DNA reference.

set (Table I). The mean number of informative $\mathrm{CpG}$ sites was 485,170 (SD 209) in the metastatic lymph nodes and 485,001 (SD 514) in the GCs without LNM ( $\mathrm{P}=0.63)$. We searched for $\mathrm{CpG}$ sites that were highly methylated in the three metastatic lymph nodes [ $\beta$ value $>$ a) 0.6, b) 0.5 and c) 0.4$]$ and hardly
Table III. Association between methylation levels of the genomic region around cg0643618 and clinical characteristics.

\begin{tabular}{lrrrr}
\hline & & \multicolumn{3}{c}{ Methylation level } \\
\cline { 3 - 5 } Parameters & $\mathrm{N}$ & Mean & SD & P-value \\
\hline Age & & & & \\
$\leq 60$ & 77 & 32.2 & 24.0 & 0.26 \\
$>60$ & 110 & 28.1 & 25.2 & \\
Gender & & & & 0.07 \\
Female & 58 & 34.9 & 26.4 & \\
Male & 129 & 27.6 & 23.6 & 0.07 \\
T category & & & & \\
T3 & 83 & 26.8 & 27.1 & \\
T4 & 96 & 33.6 & 22.5 & \\
\hline
\end{tabular}

methylated in the three primary GCs without LNM ( $\beta$ value $<0.2$ ) and the number of hypermethylated $\mathrm{CpG}$ sites was a) 1 , b) 31 and c) 209 , respectively. To obtain a practicable number of candidate $\mathrm{CpG}$ sites, we adopted a cut-off $\beta$ value of 0.5 and the $31 \mathrm{CpG}$ sites were selected for further analysis (Table II).

Selection of informative candidate genomic regions among primary GCs. Using primary GCs with and without LNM (screening set, Table I), the methylation levels of genomic regions around the $31 \mathrm{CpG}$ sites were measured by qMSP or qPTMR, which are accurate and sensitive enough to detect aberrant DNA methylation in a small population of cells. Of the 31 regions, 10 regions exhibited higher methylation levels in GCs with LNM (1.4- to 1.9-fold) than in those without LNM (Table II and Fig. 2A and B). For each of the 10 genomic regions, a cut-off methylation level was established in order that the Youden index (sensitivity + specificity - 1) would be maximized (Table II and Fig. 2).

Validation of the candidate genomic regions in a different set of samples. To validate the hypermethylation of the 10 candidate genomic regions in GCs with LNM, the methylation levels were analyzed in an independent sample set (validation set, Table I). A region around the cg06436185 CpG site revealed significantly higher methylation levels in GCs with LNM (1.5-fold) than those without $(\mathrm{P}=0.033$, Fig. $2 \mathrm{C})$, whereas the other nine regions were not validated (Table II). The region was located in the gene body of the PRKAG2 gene and did not belong to a $\mathrm{CpG}$ island (Table II). Therefore, it was unlikely that the methylation status of the region around cg06436185 affected the transcription of a gene. Using a cut-off level established in the analysis of the screening set $(28.8 \%)$, the presence of LNM was detected at a sensitivity of $43 \%$ and a specificity of $85 \%$. This result indicated that a methylation level of this region is a candidate marker for the detection of the presence of LNM.

Association between the methylation level of the genomic region around the cg06436185 CpG site and clinicopathological characteristics. Associations between the methylation level of the genomic region around cg06436185 
A

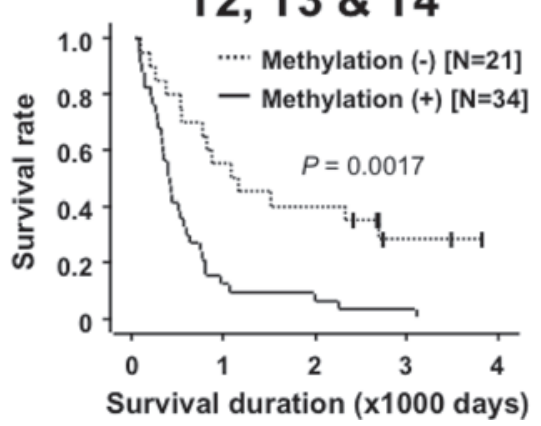

B

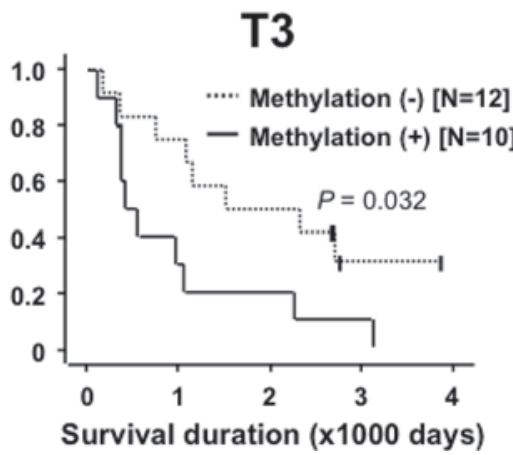

C

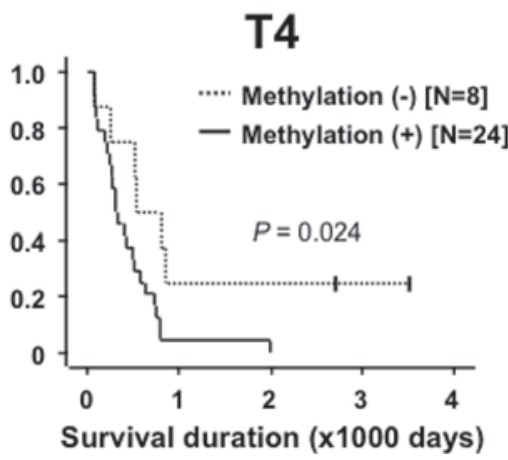

Figure 3. Kaplan-Meier analysis of 55 GC patients with LNM. (A) Overall survival in all the patients with LNM. (B and C) The survival curves of patients categorized into T3 and T4. Methylation (+), GCs with PMR of the region around cg06436185>28.8; Methylation (-), GCs with the PMR<28.8. GC, gastric cancer; LNM, lymph node metastases; PMR, percentage of the value of methylated DNA reference.

and clinicopathological characteristics (age, gender and T category) were analyzed in 157 GC patients with LNM and 30 without LNM. No difference in methylation levels according to age, gender or T category was found (Table III). Using 55 of the 157 GC patients with LNM, whose prognostic information was available (T2, one patient; T3, 22 patients; T4, 32 patients), a correlation between the methylation level and survival rate was analyzed. Patients with high methylation levels ( $>28.8 \%$; the value used to detect the presence or absence of LNM) had a significantly poorer overall survival rate compared to those with low methylation levels ( $\mathrm{P}=0.0017$; Fig. 3A). Since the T category is known to be the major prognostic factor in GC patients (26), patients in the T3 and T4 categories were analyzed separately. In the T3 and T4 subgroups, the patients with high methylation levels demonstrated a significantly poorer overall survival rate than those with low methylation $(\mathrm{P}=0.032$ and 0.024 , respectively; Fig. 3B and C). These results revealed that the high methylation level of the genomic region around $\operatorname{cg} 06436185$ was associated with an unfavorable prognosis, regardless of the depth of tumor invasion.

\section{Discussion}

Using a genome-wide methylation analysis using metastatic lymph nodes and primary GCs without LNM, a genomic region (around cg06436185) whose methylation level in primary GCs was associated with the presence of LNM was successfully identified. Notably, the association was also significant in an independent validation set $(\mathrm{P}=0.033)$. Generally, markers isolated by genome-wide analyses need to be validated in a different set of samples due to the overfitting issues caused by multiple testing (27). Even in the present study, 9 of the 10 candidate genomic regions that revealed significant hypermethylation in GCs with LNM in the screening set $(\mathrm{P}=0.0005-0.048)$ were not reproduced in the validation set. This observation emphasizes the value of the methylation level of the genomic region around $\operatorname{cg} 06436185$. Since it had a sensitivity of $43 \%$ and specificity of $85 \%$, the combined use of this novel methylation marker with imaging tools is predicted to improve the diagnostic accuracy of LNM of GCs.

The mean methylation levels of GCs with and without LNM were 18.7 and $27.5 \%$. This small difference is extremely difficult to detect by a genome-wide screening method. Our strategy in the present study was to benefit from the monoclonal growth of cells in metastatic lymph nodes and compare metastatic lymph nodes and GCs without LNM. The methylation levels of the genomic regions around $\operatorname{cg} 06436185$ were 13.2 and $54.3 \%$, respectively, in these samples. This relatively significant difference was identified using genome-wide screening, which has a relatively low accuracy in the analysis of methylation levels. Using a more accurate and sensitive method, qMSP, the small difference between GCs with and without LNM (18.7 and $27.5 \%$, respectively) was clearly demonstrated.

A method to measure methylation levels in $\mathrm{CpG}$-poor genomic regions, qPTMR, was developed using a combination of digestion with a methylation-dependent restriction enzyme and qPCR. qPTMR had an error range of 5\% in this study. It is difficult to measure methylation levels in CpG-poor genomic regions by qMSP, a well-established method with a high accuracy, due to the difficulty in designing primers. Alternatively, $M s p J I$, a recently developed methylation-sensitive restriction enzyme, recognizes ${ }^{\mathrm{m}} \mathrm{CNNR}(\mathrm{N}=\mathrm{A}, \mathrm{T}, \mathrm{G}$ or $\mathrm{C} ; \mathrm{R}=\mathrm{G}$ or $\mathrm{C})$ sequences and cleaves DNA when the $\mathrm{C}$ is methylated $(24,25)$. Since the recognition sequence is applicable to the majority of $\mathrm{CpG}$ sites and cytosines in non-CpG sites are not methylated in somatic cells, the positive cleavage by MspJI is used to determine methylation status of most CpG sites. Using qPTMR, the methylation levels of all the 19 candidate regions with few $\mathrm{CpG}$ sites were quantified. This new method is predicted to have various applications.

The methylation status of the genomic region around cg06436185 was unlikely to affect transcription of a known nearby gene (PRKAG2). However, its high methylation level in GCs, namely large fractions of cancer cells with methylation in cancer tissue, was associated with the presence of LNM and also with a poorer prognosis of the GC patients. One possible reason is that the region is located in a promoter region of unknown genes, including microRNA genes, or in enhancer regions whose methylation is critical for the regulation of gene expression levels. Another possible reason is that the methylation of the region is caused by an abnormality of unknown methylation regulation and that this abnormality is critical for tumor metsastasis or malignancy. In this case, other genomic regions are likely to be methylated in GCs with LNM or a poorer prognosis. 
In conclusion, we identified one genomic region with a methylation status in primary GCs that was associated with the presence of LNM and a poorer prognosis of GC patients.

\section{Acknowledgements}

We thank Dr Michihiro Ishida, Dr Yukie Yoda and Dr Masahiro Maeda for their assistance during sample preparation. This study was supported by the Third-term Comprehensive Cancer Control Strategy from the Ministry of Health, Labour and Welfare, Japan, by the JSPS A3 Foresight Program and by the National Cancer Center Research and Development Fund. Y.S. is a recipient of Research Resident Fellowships from the Foundation for Promotion of Cancer Research.

\section{References}

1. Matsuda A and Matsuda T: Time trends in stomach cancer mortality (1950-2008) in Japan, the USA and Europe based on the WHO mortality database. Jpn J Clin Oncol 41: 932-933, 2011.

2. Kamangar F, Dores GM and Anderson WF: Patterns of cancer incidence, mortality, and prevalence across five continents: defining priorities to reduce cancer disparities in different geographic regions of the world. J Clin Oncol 24: 2137-2150, 2006.

3. Yokota T, Ishiyama S, Saito T, et al: Lymph node metastasis as a significant prognostic factor in gastric cancer: a multiple logistic regression analysis. Scand J Gastroenterol 39: 380-384, 2004.

4. Chen JH, Wu CW, Lo SS, et al: Lymph node metastasis as a single predictor in patients with Borrmann type I gastric cancer. Hepatogastroenterology 54: 981-984, 2007.

5. Saito H, Fukumoto Y, Osaki T, et al: Prognostic significance of level and number of lymph node metastases in patients with gastric cancer. Ann Surg Oncol 14: 1688-1693, 2007.

6. Sano T and Aiko T: New Japanese classifications and treatment guidelines for gastric cancer: revision concepts and major revised points. Gastric Cancer 14: 97-100, 2011.

7. Bonenkamp JJ, Songun I, Hermans J, et al: Randomised comparison of morbidity after D1 and D2 dissection for gastric cancer in 996 Dutch patients. Lancet 345: 745-748, 1995.

8. Cuschieri A, Fayers P, Fielding J, et al: Postoperative morbidity and mortality after D1 and D2 resections for gastric cancer: preliminary results of the MRC randomised controlled surgical trial. The Surgical Cooperative Group. Lancet 347: 995-999, 1996.

9. Degiuli M, Sasako M, Ponti A, Soldati T, Danese F and Calvo F: Morbidity and mortality after D2 gastrectomy for gastric cancer: results of the Italian Gastric Cancer Study Group prospective multicenter surgical study. J Clin Oncol 16: 1490-1493, 1998.

10. Seevaratnam R, Cardoso R, McGregor C, et al: How useful is preoperative imaging for tumor, node, metastasis (TNM) staging of gastric cancer? A meta-analysis. Gastric Cancer: Aug 12, 2011 (E-pub ahead of print).
11. Ganpathi IS, So JB and Ho KY: Endoscopic ultrasonography for gastric cancer: does it influence treatment? Surg Endosc 20: 559-562, 2006

12. Yoshioka T, Yamaguchi K, Kubota K, et al: Evaluation of ${ }^{18} \mathrm{~F}$-FDG PET in patients with advanced, metastatic, or recurrent gastric cancer. J Nucl Med 44: 690-699, 2003.

13. Ha TK, Choi YY, Song SY and Kwon SJ: F18-fluorodeoxyglucosepositron emission tomography and computed tomography is not accurate in preoperative staging of gastric cancer. J Korean Surg Soc 81: 104-110, 2011.

14. Natsugoe S, Mueller J, Stein HJ, Feith M, Hofler H and Siewert JR: Micrometastasis and tumor cell microinvolvement of lymph nodes from esophageal squamous cell carcinoma: frequency, associated tumor characteristics, and impact on prognosis. Cancer 83: 858-866, 1998.

15. Kojima N, Yonemura Y, Bando E, et al: Optimal extent of lymph node dissection for $\mathrm{T} 1$ gastric cancer, with special reference to the distribution of micrometastasis, and accuracy of preoperative diagnosis for wall invasion. Hepatogastroenterology 55: $1112-1117,2008$

16. Motoyama K, Inoue H, Mimori K, et al: Clinicopathological and prognostic significance of PDCD4 and microRNA-21 in human gastric cancer. Int J Oncol 36: 1089-1095, 2010.

17. Tanaka M, Kitajima Y, Edakuni G, Sato S and Miyazaki K: Abnormal expression of E-cadherin and beta-catenin may be a molecular marker of submucosal invasion and lymph node metastasis in early gastric cancer. Br J Surg 89: 236-244, 2002.

18. Arigami T, Natsugoe S, Uenosono $\mathrm{Y}$, et al: CCR7 and CXCR4 expression predicts lymph node status including micrometastasis in gastric cancer. Int J Oncol 35: 19-24, 2009.

19. Shen Z, Ye Y, Dong L, et al: Kindlin-2: a novel adhesion protein related to tumor invasion, lymph node metastasis, and patient outcome in gastric cancer. Am J Surg 203: 222-229, 2012.

20. Bibikova M, Barnes B, Tsan C, et al: High density DNA methylation array with single $\mathrm{CpG}$ site resolution. Genomics 98: 288-295, 2011.

21. Oka D, Yamashita S, Tomioka T, et al: The presence of aberrant DNA methylation in noncancerous esophageal mucosae in association with smoking history: a target for risk diagnosis and prevention of esophageal cancers. Cancer 115: 3412-3426, 2009.

22. Niwa $\mathrm{T}$, Tsukamoto $\mathrm{T}$, Toyoda $\mathrm{T}$, et al: Inflammatory processes triggered by Helicobacter pylori infection cause aberrant DNA methylation in gastric epithelial cells. Cancer Res 70: 1430-1440, 2010.

23. Niwa T, Yamashita S, Tsukamoto T, et al: Whole-genome analyses of loss of heterozygosity and methylation analysis of four tumor-suppressor genes in N-methyl-N'-nitro-Nnitrosoguanidine-induced rat stomach carcinomas. Cancer Sci 96: 409-413, 2005

24. Zheng Y, Cohen-Karni D, Xu D, et al: A unique family of Mrr-like modification-dependent restriction endonucleases. Nucleic Acids Res 38: 5527-5534, 2010.

25. Cohen-Karni D, Xu D, Apone L, et al: The MspJI family of modification-dependent restriction endonucleases for epigenetic studies. Proc Natl Acad Sci USA 108: 11040-11045, 2011.

26. Yokota $\mathrm{T}$, Ishiyama $\mathrm{S}$, Saito $\mathrm{T}$, et al: Is tumor size a prognostic indicator for gastric carcinoma? Anticancer Res 22: 3673-3677, 2002.

27. Simon R, Radmacher MD, Dobbin K and McShane LM: Pitfalls in the use of DNA microarray data for diagnostic and prognostic classification. J Natl Cancer Inst 95: 14-18, 2003. 